\title{
Nanocrystal's Formulation of Biological Response Modifier P-MAPA on Bladder Cancer Compared to Bacillus Calmette-Guerin Immunomodulator
}

\author{
(1) Wagner José FÁVARO, Ana Claudia SILVA LIMA, (1) Queila Cristina DIAS, (1) Patrick Vianna GARCIA, \\ (1) Nelson DURÁN
}

Laboratory of Urogenital Carcinogenesis and Immunotherapy, Institute of Biology, University of Campinas (UNICAMP), Campinas-Brazil

\begin{abstract}
OBJECTIVE
P-MAPA $(200-400 \mu \mathrm{m})$ is known that exhibited significant in vivo antitumor activity, unfortunately with low capacity of suspension. Our aim is to study the effects of nanocrystals $(400-600 \mathrm{~nm})$ produced by nanonization compared to bacillus Calmette-Guerin (BCG) vaccine on bladder cancer at a non-muscle invasive stage (NMIBC).
\end{abstract}

\section{METHODS}

To induce NMIBC, 20 female Fischer 344 rats received intravesically $1.5 \mathrm{mg} / \mathrm{kg}$ dose of N-methyl-Nnitrosourea (MNU), and control animals were injected with PBS solution. After cancer induction, MNU (cancer) group received identical treatment that control group; a group received $106 \mathrm{CFU}$ dose of BCG for 6 weeks; P-MAPA-Nano+Pluronic group was injected with intravesically $0.8 \mathrm{mg} / \mathrm{kg}$ dose of P-MAPA for 6 weeks. A histopathological analysis of bladder after 16 weeks was carried out. Following the same procedure, the P-MAPA-Nano-Chitosan was studied.

\section{RESULTS}

In P-MAPA-Nano+Pluronic crystals, a better antitumor activity than BCG treatment was found and correlated with the apoptosis raised. However, unfortunately in the case of chitosan as stabilizer, the effect was negligible. One reasonable explanation to this low effect was probably through a drastic $\mathrm{pH}$ change (basic) in the urinary bladder in cancer case.

\section{CONCLUSION}

The results demonstrated that this new nanoformulation of P-MAPA in the presence of Pluronic F68 could be a potential candidate for treatment of in vivo bladder cancer.

Keywords: Apoptosis; bladder cancer; immunomodulator; P-MAPA.

Copyright $\odot$ 2022, Turkish Society for Radiation Oncology

\section{Introduction}

Urinary bladder carcer (BC) is the second most usual malignancy at urinary tract. [1-3] The American Cancer Society evaluates that there will be about 83,730 new cases of CB diagnosed in the US in 2021 (564,280 men and 19,450 women. 17,200 will be killed by this disease).[4] Over 70\% incidence of BC is superficial (pTis) and non-invasive tumor ( $\mathrm{pTa}$ and $\mathrm{pT} 1$ ), and the occurrence of invasive case is occasionally.[5] However, around $50 \%$ of non-muscle invasive tumors will died happening in 4 years after the diagnosis, and around $12 \%$ will progress to an invading phenotype.[6,7]

In general, BC treatment is performed by intravesical therapy. This treatment has the comparative advantage of decreasing systemic effects of the drugs used in

Laboratory of Urogenital Carcinogenesis and Immunotherapy, Institute of Biology,

University of Campinas (UNICAMP), Campinas-Brazil

E-mail:nelsonduran1942@gmail.com 
the treatment of urothelial tract diseases cause when administered intravenously. In general, the intravenous route requires the administration of higher doses of the drug, since a part of it is distributed throughout the body, while the other is metabolized and excreted before reaching its specific target.[8]

Besides this, the adverse effects are more pronounced. Thus, it is ideal drug delivery at the site where its action is desired, because such a strategy may increase the pharmacological efficacy and reduce drug dosage required to achieve the desired effect thus reducing the toxic effects.[8] In this direction, the intravesical route of administration of drugs in the urinary tract has already been widely used that the bladder is an organ of easy access and therefore suitable for topical therapy, in this route is possible to expose the injured area (bladder/tumors) directly to the drug and the amount of drug delivered can be easily controlled. $[9,10]$

Bladder cancer (BC), characterized by superficial lesions, has a high recurrence and progression rate after endoscopic resection. According Richie and D 'Amico[11] (2005), 70\% of bladder cancers do not invade the muscular layer of this body, yet $20-30 \%$ had progression and $70 \%$ exclusive post-treatment recurrence with transurethral resection. Moreover, it is known that intravesical instillation of bacillus Calmette-Guerin (BCG), these indices decreased to $31 \%$. [11,12] Morales et al.[13] and Morales[14] were pioneers to prove success in the treatment of superficial BC with BCG.[15] Since then, BCG is the treatment of choice for superficial $\mathrm{BC}$ of high risk and is currently considered the immunotherapy with the best results, inclusive superior to intravesical chemotherapy related to recurrence rates and tumor progression.[16] However, for patients who develop BCG unresponsive disease, cystectomy remains the standard of care.[17]

The immunotherapy with BCG results in massive immune response characterized by the induction of cytokine expression in both in urine and bladder and influx of inflammatory cells in the bladder wall. However, utilization of live-attenuated bacillus may cause side effects and complicate its prediction response. These effects are observed in $90 \%$ of patients treated with BCG and that these irritative symptoms ranging from mild to moderate urinary tract to decease, experiencing serious difficulties, such as persistent fever, hemodynamic instability, or allergic reactions difficulty its use. $[15,18,19]$ Therefore, alternative treatments using immunomodulators have been proposed with promising results. [20-25]
An immunomodulator (biological response modifier), the proteinaceous aggregate of magnesium and ammonium phospholinoleate-palmitoleate anhydride (P-MAPA) developed by Farmabrasilis from Aspergillus oryzae, [26] has appeared as an important drug for many diseases. P-MAPA exhibits anti-tumor activity in many mouse models. [27] Studies on toxicology indicated that this immunomodulator is a safe drug, through different toxicological stages carried out in rodents, monkeys, and also in human Phase I clinical trials. Besides these results, no teratogenicity effect either in vitro or in vivo studies was found. The positive effects, such as P-MAPAinduced proliferation of lymphocyte $\mathrm{T}$, rise interferon-gamma and interleukin-2, natural killer (NK) cell activity,[20-22,27-30] and promote nitric oxide (NO) delivery by macrophages were observed.[31]

A dose injected intraperitoneal (i.p) $24 \mathrm{~h}$ post-infectious confrontation on C57BL/6 mice was significantly effective and avoiding death due to Punta Toro virus infection. This administration also decreased systemic viral burden and liver anomalies on day 3 of infection. [32]

P-MAPA exhibited ligand activities as toll-like receptors (TLRs) ligand in vitro and enhanced the immunological status in bladder cancer, rising TLR2 and TLR4 protein levels. The immunotherapy by P-MAPA was efficient in restoring tumor protein (p53) and TLRs reactivity and demonstrated significant higher antitumor activity than BCG, a reference drug in these treatments. The activation of TLRs and p53 may give a possible and hypothetical mechanism for the immunotherapeutic effects in either cancer or in infectious. [33-36]

P-MAPA showed also to exhibits antileishmaniasis activity. Studies with Leishmania-infected dogs showed diminish in TLR2 in macrophages related to healthy dogs and in the case of P-MAPA's induction. Reactive oxygen species (ROS) were raised in peripheral blood mononuclear cell from Leishmania spp.-infected dogs related to healthy dogs. However, P-MAPA upgraded ROS production. NO generation was augmented in culture supernatant from macrophages promoted by P-MAPA in a similar way on healthy and Leishmania spp.-infected dogs. $[26,37,38]$ Recently was shown that immunotherapy with P-MAPA may be an alternative in the treatment of non-muscle invasive bladder cancer (NMIBC), especially in cases where BCG therapy failure, as evidenced by the effect of P-MAPA on tumor regression in comparison of BCG treatment.[39]

As the nanobiotechnology plays an important role in cancer research, [40-44] the aim of this study was to study the nanofunctionalized P-MAPA effect on bladder cancer. 


\section{Materials and Methods}

\section{Compound}

The anticancer, P-MAPA, was provided by Farmabrasilis (Sr. Iseu Nunes, Campinas, SP, Brazil).

\section{P-MAPA Characterization}

Previously, it was characterized as an aggregated magnesium and ammonium phosphate associated to linoleate-palmitoleate anhydride.[20]

\section{Nanonization of P-MAPA Microcrystals}

Nanonization of P-MAPA microcrystals was carried out in an ultrasonicator at 750 Watt (VCX 750 - Sonics). Briefly, $5 \mathrm{mg}$ of P-MAPA microcrystals were dispersed in a $5 \mathrm{~mL}$ of aqueous solution of Pluronic F68 $(5 \% \mathrm{w} / \mathrm{v})$ (Aldrich) or in $5 \mathrm{~mL}$ of a dispersion of chitosan $(0.2 \% \mathrm{w} / \mathrm{v})(105 \mathrm{kDa} \sim 81 \%$ acetylation, Polymar, Brazil). These dispersions were ultrasonicated in cycles of 10 min till 1:40 in ice bath. In each sonication cycle, the temperature of the dispersion was measured for keeping below $20^{\circ} \mathrm{C}$ during all the nanonization processes.[45,46]

\section{Characterization of the P-MAPA Nanocrystals}

\section{Scanning electron microscopy (SEM)}

The morphology was determined by SEM Jeol JSM$6360 \mathrm{LV}$ at $20 \mathrm{kV}$.

\section{$\mathrm{X}$-ray diffraction (XRD)}

The XRD was measured in a X-Diffractometer Shimadzu, model XRD7000, under radiação CuKa $(\lambda=1,54060 \AA$ ), at $40 \mathrm{kV}$, current $30 \mathrm{~mA}$, scanning at $2^{\circ} \mathrm{min}^{-1}$.

\section{Differential scanning calorimetry (DSC)}

The physical state and interaction between compounds in nanoparticles were studied by a DSC-Q10 (TA Instruments). First, samples were lyophilized and then placed in aluminum pans and sealed. All samples were heated from $10^{\circ} \mathrm{C}$ up to $160^{\circ} \mathrm{C}$, at $5^{\circ} \mathrm{C} \mathrm{min}^{-1}$ and held isothermally for $1 \mathrm{~min}$, in a nitrogen atmosphere (50 $\left.\mathrm{mL} \mathrm{min}^{-1}\right)$.

\section{Size distribution and zeta potential}

The average diameter, polydispersity index, and zeta potential were studied by dynamic light scattering technique, using a Zetasizer Nano instrument, ZEN3600 (Malvern Instruments). All samples were diluted in $1 \mathrm{mmol} \mathrm{L}^{-1}$ solution of $\mathrm{KCl}$, ratio 1:10 of sample: $\mathrm{KCl}$ solution. Measurements were performed at $25^{\circ} \mathrm{C}$. Zeta potential was measured by electrophoretic mobility using the same equipment with fixed angle of $173^{\circ}$ and a temperature of $25^{\circ} \mathrm{C}$.

\section{Influence of $\mathbf{p H}$ on the stability of the nanoparticles}

The $\mathrm{pH}$ effect on the nanoparticles size was carried out using a titrator MTP-2 Multi-Purpose Titrator $\left(\right.$ Malvern $\left.^{\circ}\right)$ coupled to the Zetasizer Malvern. Titrating solution of $\mathrm{NaOH}(0.25 \mathrm{e} 1 \mathrm{M})$ and $\mathrm{HCl}(1 \mathrm{M})$ was used. The $\mathrm{pH}$ range used was from 6 to 9 and diameter measured at intervals of $0.5 \mathrm{pH}$ 's units.

\section{In vivo Study on Bladder Cancer}

\section{Induction protocol of NMIBC}

Fisher 344 strain rats $(n=35)$ were used, at aged of 7 weeks, weighing on average 150 grams, obtained from the Vivarium of the University of Campinas (CEMIB/ UNICAMP). For the experiments, the protocol followed strictly the ethical principles in animal research (CEUA/UNICAMP-Protocol Nr 2684-1).

For induction, NMIBC used N-methyl-NNitrosourea (MNU) diluted in sodium citrate solution and administered through intravesical (Fávaro et al., 2012). Thirty animals were anesthetized with xylazine hydrochloride $2 \%$ (5 mg/kg i.m.; König, São Paulo, Brazil) and ketamine hydrochloride 10\% (60 $\mathrm{mg} / \mathrm{kg}$, i.m.; Fort Dodge, Iowa, USA), kept in this state for $45 \mathrm{~min}$ to prevent spontaneous urination, and instilled a dose of $1.5 \mathrm{mg} / \mathrm{kg}$ of N-methyl-N-Nitrosourea (MNU - Sigma, St. Louis, MO, USA) dissolved in 0.3 $\mathrm{ml}$ sodium citrate ( $1 \mathrm{M}, \mathrm{pH}$ 6) every 15 days (weeks $0,2,4$, and 6), totaling four doses (Fávaro et al., 2012). For induction procedures, we used Class II safety cabinet A2 (The Baker Company, Sanford, ME, USA). The other five animals that did not receive MNU were considered as control group (Group 1). Two weeks after MNU induction, the animals were divided into six groups (five animals per group): Group 2: MNU (cancer): Received an intravesical dose of $0.3 \mathrm{ml}$ of $0.9 \%$ saline for 6 consecutive weeks (Fávaro et al., 2012); [33] Group 3: MNU + BCG: Received intravesical dose of 106 CFU - 40 mg of BCG (Ataulpho de Paiva Foundation, Rio de Janeiro, Brazil) diluted in $0.3 \mathrm{ml}$ of saline 0.9\% for 6 consecutive weeks[33] (Fávaro et al., 2012); Group 4: MNU + P-MAP-Micro Pluronic: Received intravesical dose of $0.2 \mathrm{mg}$ P-MAP (Farmabrasilis, São Paulo, Brazil) dissolved in $0.2 \mathrm{ml}$ (concentration of $1 \mathrm{mg} / \mathrm{mL}$ ) of aqueous Pluronic F68 (5\% w/v) (Sigma-Aldrich, USA) for 6 consecutive weeks; Group 5: MNU + P-MAPA-Micro-Chitosan: Received intraves- 
ical dose of 0.2 mg P-MAPA (Farmabrasilis, São Paulo, Brazil) dissolved in $0.2 \mathrm{ml}$ (concentration of $1 \mathrm{mg} / \mathrm{mL}$ ) of aqueous chitosan $(0.2 \%)(105 \mathrm{kDa} / 81 \% \sim$ acetylation, Polymar) for 6 consecutive weeks; Group 6: MNU + PMAPA-Nano-Pluronic: Received an intravesical dose of $0.2 \mathrm{mg}$ P-MAP nanonizado dissolved in $0.2 \mathrm{ml}$ (concentration of $1 \mathrm{mg} / \mathrm{ml}$ ) aqueous solution of Pluronic F68 (5\% w/v) for 6 consecutive weeks; and Group 7: MNU+P-MAPA-Nano-Chitosan: Received an intravesical dose of $0.2 \mathrm{mg}$ P-MAPA nanonized dissolved in $0.2 \mathrm{ml}$ (concentration of $1 \mathrm{mg} / \mathrm{ml}$ ) aqueous solution of chitosan $(0.2 \%)$ for 6 consecutive weeks.

The intravesical doses in the different experimental groups were instilled through flexible catheter 20 gauge (Abocath, São Paulo, Brazil). The animals of all experimental groups received water and the same diet ad libitum (Nuvilab, Colombo, PR, Brazil). After 16 weeks of the experiment, the animals were euthanized and the urinary bladders collected and submitted to histopathological analysis.

\section{Histopathological Analyses}

Samples of the urinary bladder of all the animals of each group were collected and fixed in Bouin solution for $12 \mathrm{~h}$ and enclosed in plastic polymers (Paraplast Plus, ST. Louis, MO, USA). Then, the materials were cut on a rotary microtome Biocut 1130 (Reichert-Jung, $\mathrm{Mu}$ nich, Germany) with a thickness of $5 \mu \mathrm{m}$ and stained with hematoxylin-eosin and photographed in the light microscope Nikon Eclipse Ni-U (Nikon, Tokyo, Japan) equipped with camera Nikon DS-IR-1 (Nikon, Tokyo, Japan). The urothelial lesions were classified according to the consensus staging proposed by the World Health Organization/International Society of Urological Pathology.[47]

\section{Analysis of the Urinary $\mathrm{pH}$}

The urine samples $(200 \mu \mathrm{L})$ were collected from urinary bladder through flexible catheter 20 gauge (Abocath, São Paulo, Brazil) from all the animals in each group at the euthanasia procedure. Subsequently, the urine of each animal was applied in the $\mathrm{pH}$ uro dipstick
(Urofita 10 DLU, Prodimol Biotechnology), following the instructions provided by the manufacturer.

\section{Statistical Analysis}

Histopathological results were compared with a proportion test. The difference between the two proportions was tested using test of proportion with a type-I error of $1 \%$.

\section{Results}

\section{P-MAPA Nanoparticles Characterizations}

Nanocrystals of P-MAPA with a diameter below 500 $\mathrm{nm}$ after the nanonization process were obtained (Table 1). The surface charge was dependent of the stabilizer used. Negative charge was obtained when Pluronic F68 as used and contrary with chitosan a positive charge was observed.

These differences are explained by the structures of the stabilizers. Pluronic F68 exhibits several hydroxyl groups that give negative charge density $(\delta-)$, and in the case of chitosan, the amino group at the $\mathrm{pH}$ moiety is protonated.

Related to the morphology of the micro- and nanocrystals from P-MAPA, the former had 200-400 $\mu \mathrm{m}$ (Fig. 1a). Nanocrystals of P-MAPA stabilized by Pluronic F68 exhibited a size of $\sim 500 \mathrm{~nm}$ (Fig. 1b, c). This SEM micrograph showed agglomeration due to the Pluronic F68 presence after drying for those measurements.

The preparation of nanocrystals of P-MAPA by sonication looses completely the typical crystallinity of microparticles of P-MAPA and appeared neatly only the presence of Pluronic F68. The DSC of P-MAPA microparticles showed a peak at $\sim 122^{\circ} \mathrm{C}$ and in the presence of Pluronic F68 (nano) did not appear the P-MAPA peak by only the peak at $\sim 52^{\circ} \mathrm{C}$ corresponding to Pluronic F68. However, a DSC signal of micro P-MAPA in the presence of Pluronic F68 (8:2) ratio in a physical mixture appeared the peak at $\sim 52^{\circ} \mathrm{C}$ from Pluronic F68 and also P-MAPA peak, but at $\sim 132^{\circ} \mathrm{C}$. This shift probably was related to a different behavior of P-MAPA in Pluronic F68.

Table 1 Size and zeta potential of P-MAPA nanocrystals

\begin{tabular}{lccc} 
Samples & Diameter $(\mathbf{n m})$ & IP (polydispersity index) & Zeta potential (mV) \\
\hline P-MAPA & $300.000 \pm 100.000$ & 0.610 & $-20 \pm 4$ \\
P-MAPA-Pluronic F68 & $496.8 \pm 89.4$ & 0.541 & $-14 \pm 2$ \\
P-MAPA-Chitosan & $461.7 \pm 83.1$ & 0.573 & $+23 \pm 3$ \\
\hline
\end{tabular}

P-MAPA: Proteinaceous aggregate of magnesium and ammonium phospholinoleate-palmitoleate anhydride 

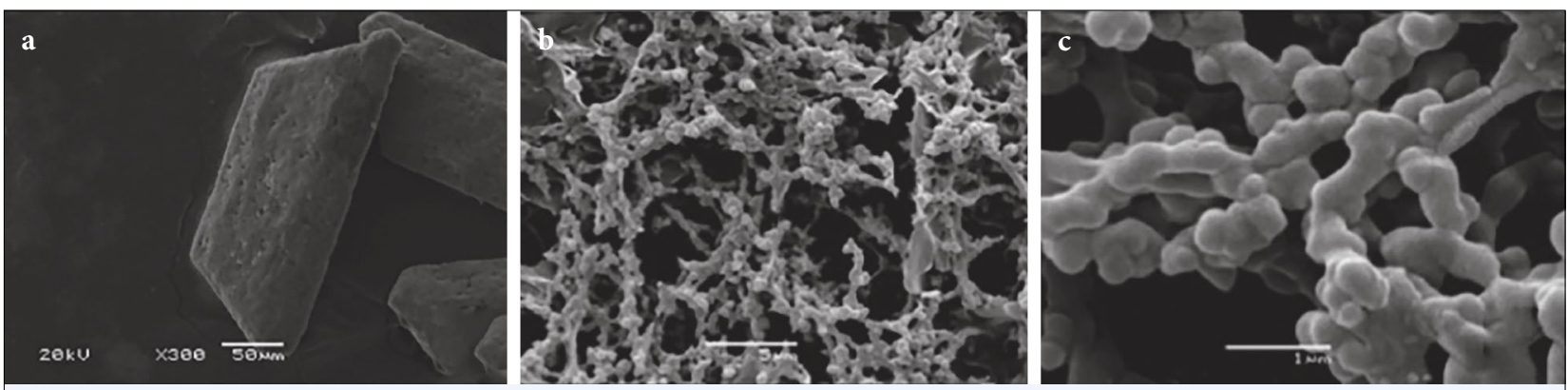

Fig. 1. (a) SEM micrograph of microcrystal of P-MAPA, (b), (c) SEM micrograph of the nanocrystals of P-MAPA stabilized by Pluronic F68.

SEM: Scanning electron microscopy; P-MAPA: Proteinaceous aggregate of magnesium and ammonium phospholinoleate-palmitoleate anhydride.

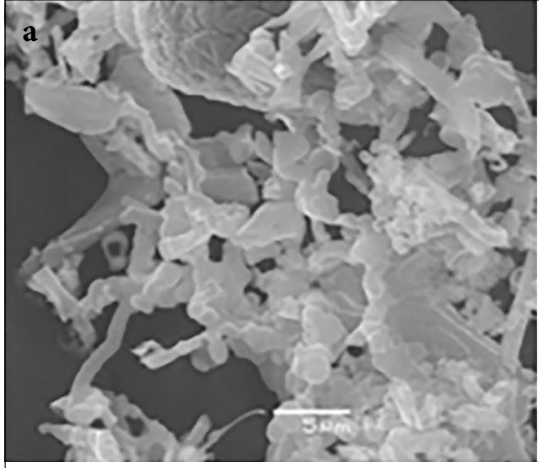

SEM of Nano-P-MAMA (water)

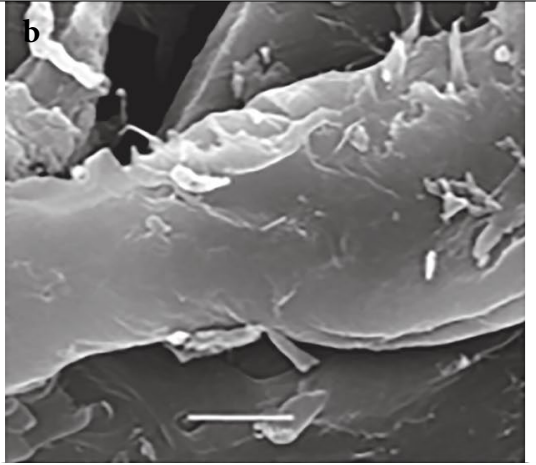

SEM of Chitosan

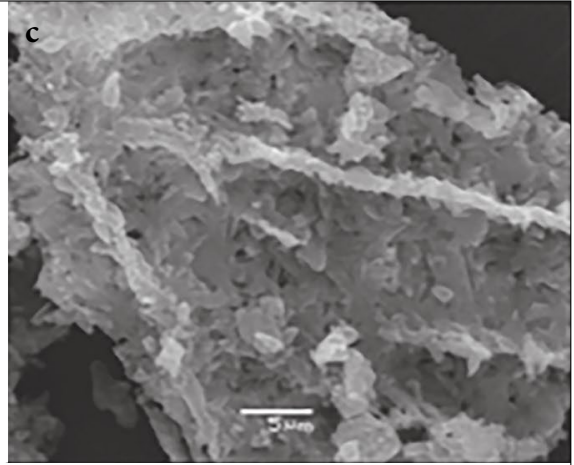

SEM of Nano-P-MAMA-Chitosan

Fig. 2. Microcrystal of P-MAPA after sonication in water with the presence of chitosan (a) SEM of nano-P-MAPA (water), DSC peak: $152 \mathrm{oC}$; (b) SEM of chitosan, DSC peak: 140oC; (c) SEM of Nano-P-MAPA in chitosan, DSC peak: $\sim 85 \mathrm{oC}$ and at $\sim 138 \mathrm{oC}$.

SEM: Scanning electron microscopy; P-MAPA: Proteinaceous aggregate of magnesium and ammonium phospholinoleate-palmitoleate anhydride.

The behavior of nanocrystal of P-MAPA in the presence of stabilizer chitosan is also different, related to Pluronic F68. Figure 2 shows that microcrystal of P-MAPA lost it crystallinity after sonication (SEM and DSC as compared with Fig. 2).

In the DSC study, mainly appears chitosan (from the DSC analysis was around $140^{\circ} \mathrm{C}$ ) presumably with some contribution of P-MAPA crystals transformation. From DSC, the loss of water around $85-90^{\circ} \mathrm{C}$ is observed, indicating different chemical structure in the presence of chitosan (not shown).

\section{Micro- and Nanoparticles of P-MAPA Stability at Different $\mathrm{pHs}$}

In the $\mathrm{pH}$ titration on nanoparticles P-MAPA, produced by Pluronic F68 as stabilizer exhibited a good range of stability (as shown by the zeta potential value at $\mathrm{pH}$ 7.5) compared to the use of chitosan as stabilizer where a great decay at the same range was observed (Fig. 3). Size distribution was not altered with Pluronic F68 at the range of pH 5-7.5 $(\sim 450 \mathrm{~nm})$ and then with a slightly increase of its size was stable at pH 7.5-9 ( 600 $\mathrm{nm})$. On contrary, the size of the nanoparticles stabilized by chitosan was quite stable at $\mathrm{pH}$ range of 5-7.5 $(\sim 500 \mathrm{~nm})$ but increased when the $\mathrm{pH}$ was more basic (over $\mathrm{pH} 7.5$ ) at to size of $3 \mu \mathrm{m}$ with significant change in the zeta potential was observed.

\section{Urinary pH}

Through urine analysis showed that the moiety presented by the urinary bladder affected by cancer exhibited basic $\mathrm{pH}$ values between 8 and 9 , and in the bladder of the control animals, the $\mathrm{pH}$ values were acids with a $\mathrm{pH}$ value between 5 and 6 . The increase in the 


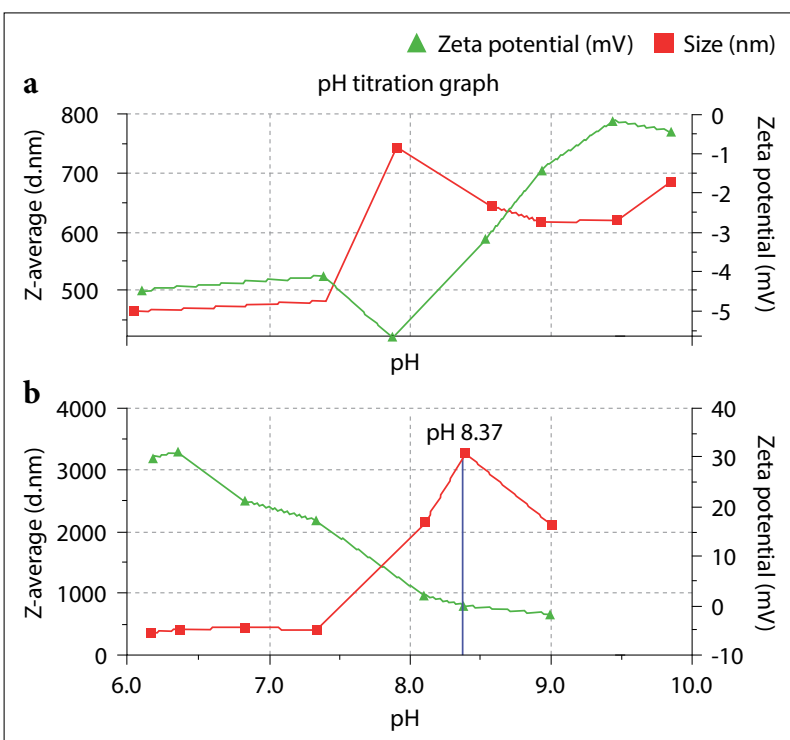

Fig. 3. Titration by $\mathrm{pH}$ effect on the size and Zeta potential of nanoparticles of P-MAPA crystals prepared by (a) Pluronic F68 and (b) Chitosan.

P-MAPA: Proteinaceous aggregate of magnesium and ammonium phospholinoleate-palmitoleate anhydride.

$\mathrm{pH}$ of the urine in NMIBC can be explained by renal impairment (hydronephrosis) detected in all animals affected by cervical cancer.

\section{Evaluation and Comparison of the Antitumoral Activity of Nanoparticles of P-MAPA Crystals with BCG Immunotherapy}

\section{Histopathological analyses}

The urinary tract control group (Group 1) showed no structural changes (Fig. 4a, b, Table 2). The normal urothelium was composed of 2-3 layers, of which basal cell layer, an intermediate cellular layer, and a surface layer composed of apical umbrella cells (Fig. 4a, b).

In contrast, urinary tract MNU group (Group 2) showed drastic histopathological changes, such as hydronephrosis and hydroureter; urothelial carcinoma invades the lamina propria (pT1) (Fig. 4c, d), papillary carcinoma (PTa), and squamous metaplasia in the bladder were found in $80 \%, 20 \%$, and $60 \%$ of animals, respectively (Table 2).

Papillary carcinoma (pTa) (40\%) (Fig. 4e), the low-grade intraurothelial neoplasia $(40 \%)$, and carcinoma in situ (pTis) were the most frequent histopathological changes in the urinary bladder of $\mathrm{MNU}$ group+BCG (Group 3) (Table 2). In addition, the urinary tract $\mathrm{MNU}+\mathrm{BCG}$ group showed significant macroscopic abnormalities such as cystic kidney lesions, hydroureter, thickening, and papillary lesions in the bladder.

The macroscopic characteristics of the urinary tract MNU group + P-MAP-Micro Pluronic (Group 4) were similar to those in the control group. In the urinary bladder, the normal urothelium was found in $20.0 \%$ of the animals (Table 2). The most frequent histopathological changes in the MNU + P-MAP-Micro Pluronic group were flat hyperplasia (60\%) (Fig. 4f) and papillary hyperplasia (20\%) (Table 2$)$.

Urinary tract MNU group + P-MAP-Micro Chitosan (Group 5) showed drastic histopathological changes, such as hydronephrosis and hydroureter; urothelial carcinoma with invasion of the lamina propria (pT1) (Fig. 4g), papillary carcinoma (pTa), carcinoma in situ (pTis), and squamous metaplasia were found in the urinary bladder in $40 \%, 40 \%, 20 \%$, and $40 \%$ of animals, respectively (Table 2 ).

The papillary hyperplasia (60\%) (Fig. 4h), the lowgrade intraurothelial neoplasia $(20.0 \%)$, and the flat hyperplasia (20\%) (Table 2) were the most frequent histopathological changes in the urinary bladder MNU group + P-MAP-Nano Pluronic (Group 6).

Similar to MNU Group, MNU group + P-MAPNano Chitosan (Group 7) showed drastic histopathological changes, highlighting papillary carcinoma (pTa) (60.0\%) (Fig. 4i, Table 2), urothelial carcinoma with invasion lamina propria (pT1) (40\%), and squamous metaplasia (60\%) (Fig. 4i, Table 2). Furthermore, changes were observed macroscopic as hydronephrosis and hydroureter.

The occurrence of urinary stones and macroscopic hematuria was observed in MNU groups, MNU + BCG, MNU + P-MAP-Micro-Chitosan, and MNU + P-MAP Nano-Chitosan. These changes were absent in $\mathrm{MNU}+\mathrm{P}-\mathrm{MAP}-\mathrm{Micro}$ Pluronic and MNU + P-MAPNano Pluronic groups.

Interesting is to compare the MNU+P-MAPAMicro Pluronic (Group 4) and MNU+P-MAPA-Nano Pluronic (Group 6), since these groups were the most effective against bladder cancer. Both groups exhibited flat hyperplasia and papillary hyperplasia, although in different distribution, only Group 4 exhibited a $60 \%$ of flat hyperplasia and $20 \%$ of normal urothelium in animal bladders. Apparently by this analysis, Group 4 looks better than Group 6. In other words, the micro P-MAPA appeared better than nano-P-MAPA in this protocol. However, in this case, it must be considered that nano-P-MAPA was around 7-fold less concentration that micro P-MAPA. 

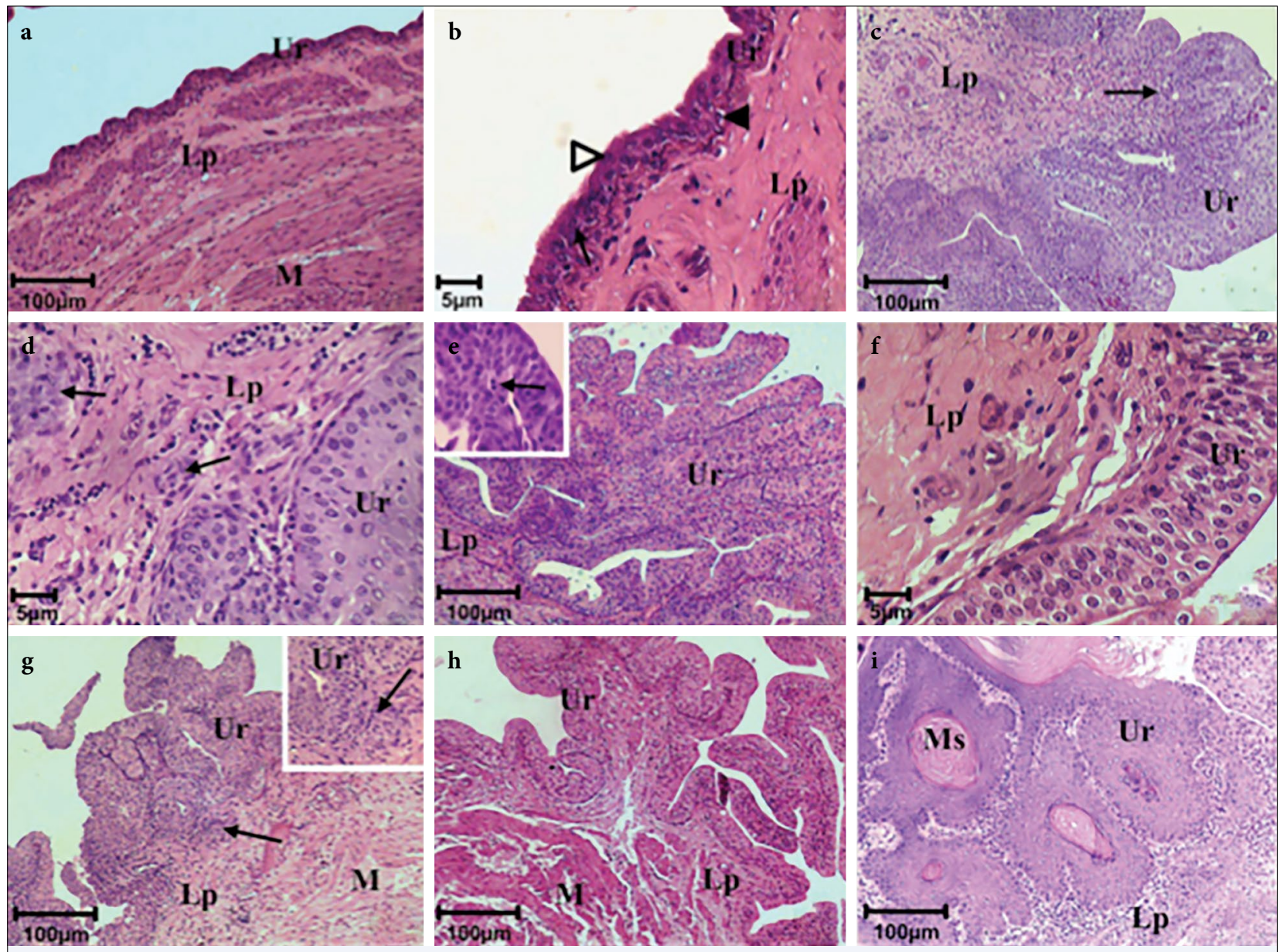

Fig. 4. Photomicrographs of the most frequently histopathological alterations of urinary bladder of control groups (a, b), MNU (c, d), MNU+BCG (e), MNU+P-MAPA-Micro Pluronic (f), MNU+P-MAPA-Micro Chitosan (g), MNU+PMAPA-Nano Pluronic (h) and MNU+P-MAPA-Nano Chitosan (i). (a), (b) Normal urothelium composed of 2-3 layers: a layer of basal cells (closed arrowhead), a middle layer of cells (arrow) and a surface layer composed of apical umbrella cells (open arrowheads). (c), (d) urothelial carcinoma with invasion of the lamina propria (pT1): neoplastic cells arranged in small groups (arrows) invading the lamina propria. (e) papillary carcinoma (pTa) characterized by extensive papillary lesions and urothelial cells with disordered arrangement and loss of polarity; and inset figure: mitotic figure (arrow). (f) Flat hyperplasia characterized by thickening of the urothelium and no atypical cytologic. (g) urothelial carcinoma with invasion of the lamina propria (pT1): neoplastic cells arranged in small groups (arrow) invading the lamina propria; inset of Fig 1g: invasion lamina propria tumor (arrow). (h) papillary hyperplasia characterized by thickening of the urothelium with papillomatosis and no atypical cytologic. (i) papillary carcinoma (pTa) associated with squamous metaplasia (Ms).

Lp: Lamina propria; M: Muscle layer; Ur: Urothelium; Ms: Squamous metaplasia; MNU: N-methyl-N-nitrosourea; BCG: Bacillus calmette-guerin; P-MAPA: Proteinaceous aggregate of magnesium and ammonium phospholinoleate-palmitoleate anhydride.

\section{Discussion}

In this study, the stabilizer Pluronic F68 showed to be more stable to $\mathrm{pH}$ variations as compared with chitosan. It was observed that the particle size of the nanoparticles of P-MAPA is not changed in basic $\mathrm{pH}$, where it remained at the nanometer scale. This fact favored treatment, because no change in the stabilizing struc- ture (Pluronic F68) ensured that its contact surface remains unchanged providing better adsorption capacity to the urothelium of the bladder and consequently internalization of the P-MAPA. This can be seen because the structure of the P-MAPA and Pluronic F68 was a eutectic mixture, even at low concentration of Pluronic F68 such as at $0.3 \%$, as demonstrated by DSC and by the diffraction in the XRD measurements. 
Table 2 Percentage of histopathological alterations in the urinary bladder of the animals from control groups (Group 1),
MNU (Group 2), MNU+BCG (Group 3), MNU+P-MAPA-Micro Pluronic (Group 4), MNU+P-MAPA-Micro-Chitosan
(Group 5), MNU+P-MAPA-Nano Pluronic (Group 6), and MNU+P-MAPA-Nano-Chitosan (Group 7)

Groups

Histopathology

\begin{tabular}{ccccccc}
\hline $\begin{array}{c}\text { Group 1 } \\
(n=5)(\%)\end{array}$ & $\begin{array}{c}\text { Group 2 } \\
(n=5)(\%)\end{array}$ & $\begin{array}{c}\text { Group 3 } \\
(n=5)(\%)\end{array}$ & $\begin{array}{c}\text { Group 4 } \\
(n=5)(\%)\end{array}$ & $\begin{array}{c}\text { Group 5 } \\
(n=5)(\%)\end{array}$ & $\begin{array}{c}\text { Group 6 } \\
(n=5)(\%)\end{array}$ & $\begin{array}{c}\text { Group 7 } \\
(n=5)(\%)\end{array}$
\end{tabular}

Normal

Flat hyperplasia

Papillary hyperplasia

Low-grade intraurothelial neoplasia

High-grade intraurothelial neoplasia-

Carcinoma in situ (pTis)

Papillary carcinoma (pTa)

Urothelial carcinoma with invasion

of lamina propria (pT1)

$\begin{array}{ccccccc}05(100)^{*} & - & - & 01(20) & - & - & - \\ - & - & - & 03(60)^{*} & - & 01(20) & - \\ - & - & - & 01(20) & - & 03(60)^{*} & - \\ - & - & 02(40)^{*} & - & - & 01(20) & - \\ - & - & 01(20)^{*} & - & 01(20)^{*} & - & - \\ - & 01(20) & 02(40) & - & 02(40) & - & 03(60)^{*} \\ - & 04(80)^{*} & - & - & 02(40) & - & 02(40)\end{array}$

The histopathological alterations are expressed as a percentage of the number of rats $(n)$ examined in each group. ${ }^{*} \mathrm{P}<0.0001$ (proportions test). Benign lesions: Flat hyperplasia; Pre-neoplastic lesions: Low-grade intraurothelial neoplasia; Neoplastic lesions: pTis, pTa, and pT1. MNU: N-methyl-N-nitrosourea; BCG: Bacillus calmette-guerin; P-MAPA: Proteinaceous aggregate of magnesium and ammonium phospholinoleate-palmitoleate anhydride

Regarding chitosan, it can be concluded that the basic $\mathrm{pH}$ of the urine in the urinary bladder led to deprotonation and precipitation of chitosan that hindered the adhesion and action of P-MAPA in the urothelium, resulting in low effectiveness of this drug in the treatment of intravesical NMIBC. The low affectivity of chitosan as stabilizer of nanoparticles of P-MAPA is possible to explains in the following manner: The zeta potential value of the nanonized P-MAPA with chitosan was positive $\mathrm{pH} 6$ which decreases as the $\mathrm{pH}$ becomes neutral or basic, reflecting the deprotonation of chitosan terminal, indicating that the electrostatic surface charges on the colloidal have been eliminated. This effect can be illustrated schematically (Fig. 5).

The $\mathrm{pH}<6$, the amino groups of chitosan are fully protonated and the P-MAPA chitosan is individually dispersed in water due to strong electrostatic repulsion. Increasing the size can be explained due to the chitosan terminals are extended and the semi-rigid structure. At the $\mathrm{pH} \sim 7.5$, most chitosan segments are deprotonated and intramacromolecular and internal repulsion decreases, resulting in twisting of the chitosan chains. However, Columbic residual forces prevailing overwhelm the hydrogen bond between the domains of $\mathrm{P}$ MAPA and chitosan, preventing the formation of larger aggregates, which explain the maintenance of the size in this $\mathrm{pH}$ range. The $\mathrm{pH}>7.5$ apparently begins to neutralize the P-MAPA-chitosan structure and gradually begins to aggregate and precipitate due to hydrophobic association and the strong binding interaction with hydrogen. Similar effects were found with graphene oxide and chitosan. [48-51] Therefore, the understanding of
$\mathrm{pH}$ is critical for the application of formulations containing chitosan.

The previous studies in our laboratories have demonstrated that microparticles P-MAPA intravesically used at a dose of $5 \mathrm{mg} / \mathrm{kg}$ (concentration of $6.25 \mathrm{mg} / \mathrm{mL}$ ) produced a significant antitumor effect in NMIBC. [52] In this study, the nanoparticles of P-MAPA were administered at a dose of $1 \mathrm{mg} / \mathrm{mL}$, that is, a reduction in the dose of 6.25 times the dose used initially. Even with this reduction, stabilization of micro and Pluronic P-MAPA nanocrystals ensured the similar antitumor effect of this drug observed at the dose of $6.25 \mathrm{mg} / \mathrm{mL}$. The stabilized PMAPA with Pluronic allowed greater suspension and reducing the therapeutic dose of P-MAPA, and ensures that their contact surfaces remain unchanged providing better adsorption capacity to the bladder urothelium

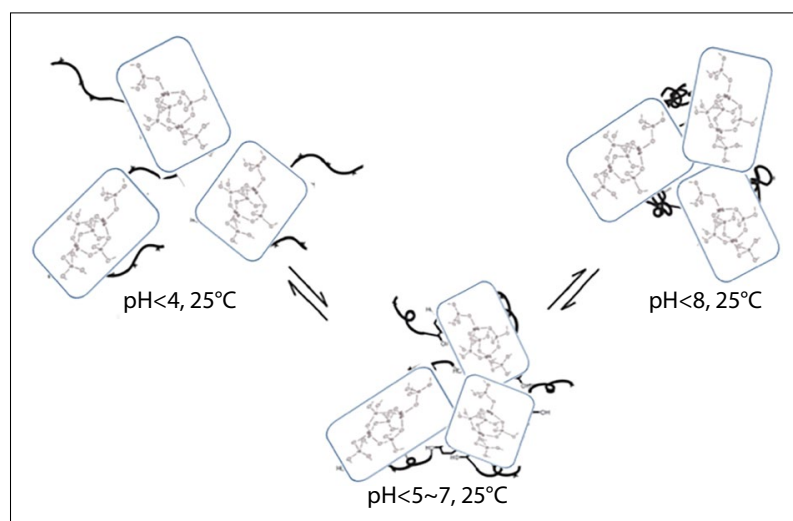

Fig. 5. Deprotonation scheme of the amino terminal group of chitosan (modified from [48]). 
and therefore greater effectiveness in the treatment of immunotherapy when compared NMIBC by BCG.

\section{Conclusion}

Taking in account these present available data, this new nanoformulation of P-MAPA immunotherapy in the presence of Pluronic F68 could be a potential candidate for treatment of patients with non-muscle invasive bladder cancer.

Peer-review: Externally peer-reviewed.

Conflict of Interest: All authors declared no conflict of interest.

Ethics Committee Approval: The study was approved by The Animal Use (CEUA) of University of Campinas Ethics Committee (No: 2684-1, Date: 04/11/2012).

Financial Support: This study has received support by São Paulo Research Foundation, FAPESP/ Brazil (grants 12/16880-7, 12/11002-1).

Authorship contributions: Concept - W.J.F., N.D., A.C.S.L.; Design - W.J.F., N.D., A.C.S.L.; Supervision - W.J.F., N.D.; Funding - W.J.F., N.D., A.C.S.L.; Materials - A.C.S.L., Q.C.D., P.V.G.; Data collection and/or processing - A.C.S.L., Q.C.D., P.V.G.; Data analysis and/or interpretation - W.J.F., N.D., A.C.S.L., Q.C.D., P.V.G.; Literature search - W.F.J., N.D., A.C.S.L., P.V.G.; Writing - W.J.F., N.D., A.C.S.L.; Critical review - W.J.F., N.D., A.C.S.L., Q.C.D., P.V.G.

\section{References}

1. Lenis A T, Lec PM, Chamie K. Bladder cancer: A review. JAMA 2020;324(19):1980-91.

2. Patel VG, Oh WK, Galsky MD. Treatment of muscleinvasive and advanced bladder cancer in 2020. CA: A Cancer J Clinic 2020;70(5):404-23.

3. Dobruch J, Oszczudłowski M. Bladder cancer: current challenges and future directions. Medicina 2021;57(8):749.

4. American Cancer Society-2021. Available at: https:// www.cancer.org/cancer/bladder-cancer/about/key-statistics.html. Accessed Jan 26, 2022.

5. Ottley EC, Pell R, Brazier B, Hollidge J, Kartsonaki C, Browning L, et al. Greater utility of molecular subtype rather than epithelial-tomesenchymal transition (EMT) markers for prognosis in high-risk non-muscle-invasive (HGT1) bladder cancer. J Pathol Clin Res 2020;6(4):238-51.

6. Shimada K, Fujii T, Anai S, Fujimoto K, Konishi N. ROS generation via NOX4 and its utility in the cytological diagnosis of urothelial carcinoma of the uri- nary bladder. BMC Urol 2011;11:22.

7. Jager L, Choy B. Cytology-neoplastic. https://www. pathologyoutlines.com/topic/bladdercytologyneoplastic.html. Accessed Oct 13, 2021.

8. Cheng C, Qiu D, Chen J, Zu X, Liu J, Li H, et al. Efficacy of intra-arterial plus intravesical chemotherapy for high risk non-muscle-invasive bladder cancer: A pooled analysis. Front Pharmacol 2021;12:707271.

9. Adiyat KT, Katkoori D, Soliwan MS. Intravesical chemotherapy. In: Lokeshwar VB, Merseburger SH, Hautmann SH, editors. Bladder tumors: molecular aspect and clinical management. New Jersey: Humana Press; 2010. p. 253-70.

10. Liu K, Zhu J, Song Y-X, Wang X, Zhou K-C, Lu Y, et al. Thermal intravesical chemotherapy reduce recurrence rate for non-muscle invasive bladder cancer patients: A meta-analysis. Front Oncol 2020;10:29.

11. Richie JP, D’Amico A. Urologic oncology. 1st ed. Philadelphia: Elsevier Saunders; 2005. p. 810.

12. Matulewicz RS, Steinberg GD. Non-muscle-invasive bladder cancer: overview and contemporary treatment landscape of neoadjuvant chemoablative therapies. Rev Urol 2020;22(2):43-51.

13. Morales A, Eidinger D, Bruce AW. Bacillus CalmetteGuerin in the treatment of adenocarcinoma of the kidney. J Urol 1976;115(4):377-80.

14. Morales A. BCG: A throwback from the stone age of vaccine opened the path for bladder cancer immunotherapy. Can J Urol 2017;24(3):8778-93.

15. Guallar-Garrido S, Julián E. Bacillus Calmette-Guérin (BCG) therapy for bladder cancer: An update. Immuno Targets Ther 2020;9:1-11.

16. Andreas BO, Brandau S. Immune mechanisms in bacillus Calmette Guerin immunotherapy for superficial bladder cancer. J Urol 2003;170(3):964-9.

17. Bree KK, Brooks NA. Kamat AM. Current therapy and emerging intravesical agents to treat non-muscle invasive bladder cancer. Hematol/Oncol Clin North Am 2021;35(3):513-29.

18. Bhole A, Brandau S. Immune mechanisms in bacillus Calmette Guerin Immunotherapy for superficial bladder cancer. J Urol 2003;170(3):964-9.

19. Lobo N, Brooks NA, Zlotta AR, Cirillo JD, Boorjian S, Black PC, et al. 100 years of Bacillus Calmette-Guérin immunotherapy: from cattle to COVID-19. Nature Rev Urol 2021;18:611-22.

20. Durán N, Nunes ODS. New polymer of proteic magnesium ammonium phospholinoleate anhydride from Penicillum sp (PB-73 Strain). Braz J Med Biol Res 1990;23(12):1289-302.

21. Durán N, Haun M, Da Silva L P, Pisani R, Pisani F J, Souza-Brito AR, et al. Comparison of the antiviral activity and toxicity of a protein magnesium ammonium phospholinoleate anhydride polymer with other antivi- 
ral drugs. Braz J Med Biol Res 1990;23(12):1303-13.

22. Durán N, Justo GZ, Queiroz MLS, Viera-Matos AN, Rettori O. New perspective in immunomodulatory therapy of tumor induced by an extracelular aggregated polymer isolated from Aspergillus orizae. Inter J Mol Med 1999;4(Suppl 1):S49.

23. Perabo FG, Willert PL, Wirger A. Superantigen-activated mononuclear cells induce apoptosis in transitional cell carcinoma. Anticancer Res 2005;25(5):356573.

24. Sanl $\mathrm{O}$, Lotan Y. Alternative therapies in patients with non-muscle invasive bladder cancer. Turk J Urol 2017;43(4):414-24.

25. Lebacle CX, Loriot Y, Irani J. BCG unresponsive high grade non muscle invasive bladder cancer: what does the practicing urologist need to know? World J Urol 2021;39:4037-46.

26. Durán N, Fávaro WJ. Biogenic synthesis of magnesium phosphate derivatives: A review on their uses and applications. Quim Nova 2018;41(5):567-76.

27. Justo GZ, Durán N, Queiroz MLS. Natural killer cell activity, lymphocyte proliferation and cytokines profile in tupour-bearing mice treated with MAPA, a agnesium aggregated polymer from Aspergillus orizae. Immunopharmacol Immunotoxicol 2003;25(3):30519.

28. Justo GZ, Durán N, Queiroz MLS. Effect on splenic T lymphocytes in Ehrlich ascites tumor bearing mice induced by MAPA - role of cytokines. Inter J Mol Med 1999;4 (Suppl 1):S49.

29. Justo GZ, Durán N, Queiroz MLS. Myelopoietic response in tumor-bearing mice by an aggregated polymer isolated from Aspergillus orizae. Eur J Pharmacol 1999;388(3):219-26.

30. Melo A, Justo GZ, Queiroz MLS. Stimulation of myelopoiesis in Listeria monocytogenes-infected mice by an aggregated polymer isolated from Aspergillus oryzae. Human Exper Toxicol 2001;20(1):38-45.

31. Bromberg N, Justo GJ, Seabra AB, Durán N. Macrophage nitric oxide (NO) stimulation by an immunomodulator: P-MAPA. Nitric Oxide-Biology and Chemistry 2006;14(4):A37.

32. Durán N, Gowen BB, Costa FTM, Justo GZ, Brocchi $\mathrm{M}$, Nunes OS, et al. A biotechnological product and its potential as a new immunomodulator for treatment of animal phlebovirus infection: Punta Toro virus. Antiviral Res 2009;83(2):143-7.

33. Fávaro WJ, Nunes OS, Seiva FRF, Nunes IS, Woolhiser LK, Durán N, et al. Effects of P-MAPA immunomodulator on Toll-like receptors and p53: Potential therapeutic strategies for infectious diseases and cancer. Infect Agents Cancer 2012;7(1)14.

34. Garcia PV, Apolinário LM, Böckelmann PK, Nunes IS, Durán N, Fávaro WJ. Alterations in ubiquitin ligase
Siah-2 and its corepressor N-CoR after P-MAPA immunotherapy and anti-androgen therapy: new therapeutic opportunities for non-muscle invasive bladder cancer. Inter J Clin Exper Pathol 2015;8(5):4427-43.

35. Garcia PV, Seiva FRF, Carniato AP, de Mello Júnior W, Durán N, Macedo AM, et al. Increased toll-like receptors and p53 levels regulate apoptosis and angiogenesis in non-muscle invasive bladder cancer: mechanism of action of P-MAPA biological response modifier. BMC Cancer 2016;16:422.

36. Socca EAR, Reis SK, Genaro SC, Leite SK, Reis IB, Bockelmann PK, et al. P-MAPA, a promisor immunomodulator against tumor cells of colonic tissues: An investigation of the action mechanism over the TLR4 signaling pathway. Life Sciences 2020;242(10):117185.

37. Santiago MEB, Silveira Neto E, Costa A, Munari DP, Andrade MMC, Somenzari MA, et al. Improvement in clinical signs and cellular immunity of dogs with visceral leishmaniasis using the immunomodulator PMAPA. Acta Tropica 2013;127(3):174-80.

38. Melo LM, Perosso J, Almeida BFM, Silva KLO, Somenzari MA, de Lima VMF. Effects of P-MAPA immunomodulator on Toll-like receptor 2, ROS, nitric oxide, MAPKp38 and IKK in PBMC and macrophages from dogs with visceral leishmaniasis. Inter Immunopharmacol 2014;18(2):373.

39. Fávaro WJ, Böckelmann PK, Garcia PV, Socca EA, Durán N. Effects of P-MAPA as immunomodulator on markers for energy metabolism in bladder cancer. Ann Urol Oncol 2020;3(1):27-35.

40. Ingle AP, Gupta I, Durán N, Rai M. Nanotherapy: A next generation hallmark for combating cancer. In: Ficai A, Grumezescu AM, editors. Nanostructures for cancer therapy. Amsterdam: Elsevier Amsterdam; 2017. p. 811-24.

41.Da Silva RC, de Souza JG, Seabra AB, Durán N, Fávaro WJ. Advances and perspectives in urinary bladder cancer nanotherapy. Eur Med J Urol 2018;6(1):52-61.

42. Durán N, Fávaro WJ. Nanopharmaceuticals and their applications in bladder cancer therapy: A mini review. J Braz Chem Soc 2018;29(5):973-81.

43. Durán N, Fávaro, WJ. Immunomulators acting on Covid-19: Actual knowledge and perspectives. Preprints. 2020 Jul 5. Doi: 10.20944/preprints202007.0090.v1. [Epub ahead of print].

44. Barani M, Hosseinikhah SM, Rahdar A, Farhoudi L, Arshad R, Cucchiarini M, et al. Nanotechnology in bladder cancer: Diagnosis and treatment. Cancers 2021;13(9):2214.

45. Durán M, Marcato PD, Tasic L, Durán N. Nanonization of biotechnological product: Nanocrystals and its polymorphisms Proceedings of 7th Congress SBPMat. Guaruja, SP, Brazil 2008; 7: Abst. H588

46. Lima ACS. Comparative study of the immunomod- 
ulator effects of P-MAPA encapsulated in mucoadhesive polymeric nanoparticles versus BCG immunotherapy in progression of non-muscle invasive urinary bladder cancer. PhD thesis at UNESP. Botucatu, Brazil; 2016.

47. Epstein JI, Amin MB, Reuter VR, Mostofi FK. The World Health Organization/International Society of Urological Pathology consensus classification of urothelial (transitional cell) neoplasms of the urinary bladder. Bladder Consensus Conference Committee. Am J Surg Pathol 1998;22(12):1435-48.

48. Bao H, Pan Y, Ping Y, Sahoo NG, Wu T, Li L, et al. Chitosan-functionalized graphene oxide as a nanocarrier for drug and gene delivery. Small 2011;7(11):156978.

49. Karki N, Tiwari H, Tewari C, Rana A, Pandey N, Basak
$\mathrm{S}$, et al. Functionalized graphene oxide as a vehicle for targeted drug delivery and bioimaging applications. J Mat Chem B 2020;8(36):8116-48.

50. Sattari S, Adeli M, Beyranvand S, Nemati M. Functionalized graphene platforms for anticancer drug delivery. Inter J Nanomed 2021;16:5955-80.

51. Shu M, Gao G, Zeng M, Yu C, Wang X, Huang R, et al. Microwave-assisted chitosan-functionalized graphene oxide as controlled intracellular drug delivery nanosystem or synergistic antitumour activity. Nanoscale Res Lett 2021;16:75.

52. Fávaro WJ, Billis A, Nunes IS, Durán N. New immunotherapy for non-muscle invasive bladder cancer (NMIBC): effects of immunomodulator P-MAPA. J Urol 2012;187(4S):e231. 